\title{
Confabulations after Bilateral Consecutive Strokes of the Lenticulostriate Arteries
}

\author{
Antonio Carota ${ }^{a, b}$ Pasquale Calabrese ${ }^{c, d}$ \\ ${ }^{a}$ Hildebrand Clinic, Rehabilitation Center, Brissago, ${ }^{b}$ Service of Neurology, \\ CHUV Hospital, Lausanne, 'Department of Psychology, Basel University, Basel, \\ and ${ }^{\mathrm{d}}$ Neurocenter of Southern Switzerland, Lugano, Switzerland
}

\section{Key Words}

Confabulation - Delusion - Lenticulostriate arteries - Anterograde memory · Caudate nucleus $\cdot$ Stroke

\begin{abstract}
We describe the case of a 75-year-old woman who manifested persistent confabulations after two consecutive strokes encompassing the area of the lenticulostriate arteries territory on both hemispheres. Findings reported on this rare clinical syndrome suggest that fantastic confabulations and delusional thoughts may arise after bilateral damage of subcortical nonthalamic structures.
\end{abstract}

\section{Introduction}

Confabulations could be defined as false memories that are believed to be true. However, this definition probably not only applies to different symptoms and hence deserves further classification [1,2] (for example, the distinction between spontaneous and provoked confabulations) but also appeals to a broader spectrum of disorders (psychosis, delirium, Korsakoff disease, stroke) and to multiple brain underpinnings.

Confabulatory syndromes arising from focal stroke and confined to specific brain areas are not infrequent. They include the 'corporeal' confabulations associated with anosognosia of hemiplegia, hemianopia or blindness after stroke including strategic associative areas on the right hemisphere [3], delusional misidentification syndromes with right frontotemporal strokes [4], 'spontaneous' or 'provoked' confabulations after stroke within the frontal-subcortical circuits processing both executive functions and memory [the limbic thalamus (dorsomedial and anterior nuclei) [5], and the orbitofrontal cortex (with rupture of aneurysms of the anterior communicating artery) [6]]. 
Confabulatory syndromes arising after focal ischemic damage of subcortical nonthalamic areas are exceptional. We describe the case of a patient who showed persistent confabulations after two consecutive strokes of the territory of the lenticulostriate arteries on both hemispheres.

\section{Case Report}

A 75-year-old right handed Swiss woman, with 12 years of education, was transferred to the rehabilitation center 1 month after a right ischemic subcortical stroke, which manifested with delirium and transitory left facial-brachial paresis.

A cardioembolic etiology was retained as the patient had chronic atrial fibrillation and the anticoagulation rate was below the therapeutic range. Four years before, she had a left lenticulostriate artery territory stroke with behavioral symptoms (confabulations and subcortical aphasia), which completely recovered after 2 weeks. The patient was a retired housekeeper who lived autonomously with no history of alcohol consumption, nutritional disorders or memory disturbances before the actual stroke (IQCODE under the cutoff score for dementia).

Brain MRI, performed at 6 weeks after the onset of the last stroke, showed the two lesions: a subacute ischemic lesion in the territory of the right lenticulostriate artery (involving the caudate head, putamen and part of the pallidum), and an ancient lesion in the territory of the left lenticulostriate artery (involving the caudate head and the surrounding white matter with the ipsilateral frontal horn enlargement) (fig. 1). No significant MRI atrophy patterns suggested degenerative dementia.

Neurological examination showed prominent behavioral and cognitive changes, without motor or sensory signs.

\section{General Behavioral and Cognitive Assessment}

The patient's language was characterized by a continuous stream of spontaneous confabulations and delusional thoughts. Each time, without relevance to the context, either with staff or visitors, the patient provided unrequested and unrealistic accounts on a variety of topics. Some of them were recurrent as 'I have a plant that is growing in the intestine because of the dietary supplements, which I am taking on medical advice. I saw that plant the first time in my bathroom at home'. Other ones occurred isolated as the marriage proposals from therapists, imaginary visits by relatives and friends, late at night always in bizarre circumstances and fantastic scenarios. In one occasion, she said to one of the examiners that, 'few hours before, a human voice originating from the near lake suggested me to behave affably with your brother, whom I knew personally some years before'. On another circumstance the patient claimed that the brother of a famous Italian politician had recently deceased, and that the patient's sister was right at that moment traveling to Rome by train for the funerals, dressed with religious clothes. Such statements generally withstood against contradictions offered by the medical staff: faced to the evidence (on the journals or TV) that the brother of the Italian politician had not died at all, she stated that the press was not updated yet. However, despite high emotional expression, only occasionally the patient seemed to behave according to the content and facts of the confabulations, giving the impression to be interested only in the verbal reports of such random thoughts.

These confabulations persisted over the 6 weeks of hospitalization and until the 6 months of follow-up, despite several behavioral interventions and pharmacological treatments (SSRIs, neuroleptics, acetylcholine-esterase inhibitors).

Standard neuropsychological assessment (table 1) showed deficits in orientation to time and personal information, sustained attention, executive functions (reduced mental flexibility, perseverative behavior, compromised control on automatic responses, incapacity of planning) and a severe anterograde memory impairment in memory tests. However, there were some residual episodic learning capacities (as documented by the Galveston Orientation and Amnesia Test, according to which the patient had a borderline performance, and by the RAVLT delayed recall score which showed a partial long-term retention). This was also reflected by the fact that the patient was 
able to recognize names and faces of therapists, and with a little practice she learned and remembered the route from the offices back to her room. Temporal orientation significantly improved in the last period of rehabilitation.

Language and visuoperceptive skills were slightly affected: conversation was fluent without paraphasias; comprehension of test instructions and object recognition (including overlapped or degraded figures) were good.

A confabulation battery [7] showed that confabulations were triggered by episodic memory cues and 'do not know' questions (either episodic or semantic), as usually found for confabulators [1], and not upon personal semantic and linguistic cues.

\section{Discussion}

After two temporally separated, bilateral consecutive strokes in the lenticulostriate artery territories the patient showed, over at least 6 months, a deep and persistent behavioral change consisting of spontaneous confabulations and delusions associated with a severe impairment of the episodic memory. This clinical picture could not be explained uniquely by an attention deficit as in delirium (e.g. forward span in the normal range and attention improvement in the early follow-up, absence of cognitive and vigilance fluctuations). Hence, it should be considered as a dementia after strategic strokes. Furthermore, and most importantly, the persistent confabulations and delusions dominated the clinical picture, precluding most rehabilitative efforts.

Persistent confabulations after localized bilateral caudate stroke should be considered an extremely rare clinical pattern as bilateral caudate strokes are rare and similar case studies were not previously reported. The pervasiveness and persistence of confabulations are probably related to the bilaterality of the lesions. The bilateral interruption of frontal-subcortical circuits (and more specifically the connections of the caudate nucleus) might be the putative mechanism of the behavioral changes [8]. Similar to thalamic post-stroke amnesia [9] or Wernicke-Korsakoff disease [10], where memory deficits and confabulations arise after damage of mammillo-thalamic tracts or thalamic nuclei, it might be speculated that in our case, again, the bilaterality of damage (and thus precluding compensatory mechanisms) is the crucial factor leading to the persistence of the syndrome.

For our patient, the peculiar aspects of the confabulation syndrome consisted of: the spontaneous character, the pervasiveness with bizarre concatenation of unrelated contents and ideas ('mosaic confabulations'), the presence of fantastic, visionary or delusional themes with emotional involvement and weak autobiographical references, the association with severe executive dysfunction and anterograde memory deficits. Within confabulatory syndromes, a distinction has been proposed between 'provoked' and 'spontaneous' confabulation [1], although this dichotomy is subject of founded criticisms [7].

The patient's confabulations might fit better with the category of spontaneous confabulation as they consisted mostly of 'incoherent and context-free retrieval of memories and associations' [1]. However, as in provoked confabulations, she showed prominent intrusions and irrelevant memories during retrieval attempts in challenge to anterograde memory tests. Furthermore, her confabulations showed all the general peculiarities that, according to Dalla Barba [7], are common to all the variants of the 
confabulatory syndromes: (1) general memories, habits and misplacements, (2) memory confusion, and (3) autoreferential contamination.

According to Kopelman's view [11], spontaneous confabulations result from the superimposition of frontal dysfunction on an organic amnesia (although this pattern of cognitive impairment may also be present in patients with provoked confabulations), while Dalla Barba and Boisse [1] emphasize the presence of different lesion sites (within the frontal lobe systems) and the integrity of the medial temporal lobe. To other authors, confabulations could reflect retrieval of irrelevant past memories [6] or overlearned information in patients with shallow encoding strategies [12].

The latter line of argumentation is also followed by Moscovitch and Melo [13] who argued that confabulations result from a failure in the 'strategic retrieval processes' involved in focusing the memory search and in monitoring the veracity of retrieved memories implying that a monitoring deficit is necessary for confabulation to occur.

Another view suggests that confabulations reflect the inability to distinguish a memory derived from the direct experience from one generated by imaginative acts [14], or the inability to handle ambiguous or metaphoric cues in thoughts or speech [15]. According to others, emotional factors might be the most relevant mechanisms for confabulations to provide more positive self-representations than true memories and to protect from unacceptable aspects of the reality [16].

Although all these mechanisms can be advocated together for the confabulations found in our patient, they also share, together with the pattern of cognitive deficits (attention, memory and executive deficits), many similarities with the thought disorder exhibited by patients with schizophrenia ('pseudologia fantastica'). These similarities refer to the reduced communicability, the fragmentation, discontinuity and incoherence of arguments, the creation of false memories as well as perceptions or beliefs about the self or the environment. Other similarities include the arousal of negative emotions, the idiosyncratic symbolization of a visionary world (where nonhuman objects live), and the interpretation of fantastic scenarios as if the patients were governed by random external stimuli.

This single-case study offers further evidence that different types of confabulations may arise even after bilateral damage of subcortical structures because of the probable disconnection of prefrontal and limbic regions involved in episodic memory processing, affect and mood regulation. These 'subcortical' spontaneous confabulations after bilateral caudate involvement seem to differ from the spontaneous confabulations due to ventromedial and orbitofrontal lesions [6], since they seem to have only weak connections with past real memories that should fail to undergo an appropriate reality monitoring.

Our case study indicates that fantastic or implausible confabulations after bilateral caudate nuclei involvement seem to share many similarities with delusions of schizophrenic patients [17] and with psychotic-like symptoms infrequently reported in patients with Huntington's disase $[18,19]$. This 'caudate' confabulatory syndrome could still be the result of the damage of several interconnected diffusely distributed fronto-limbic cortico-subcortical circuits and might correspond more to an emotionbased models of delusions [15]. 


\section{Acknowledgement}

The authors thank Dr. A. Valente for his insightful and constructive contribution to the manuscript. 
Table 1. Summary of neuropsychological assessment*

\begin{tabular}{lcl}
\hline Test & Raw score & $\begin{array}{l}\text { Perfor- } \\
\text { mance } \\
\text { rating }\end{array}$ \\
& & \\
\hline Conceptual & & $\downarrow \downarrow$ \\
MMSE & $19 / 30$ & $\downarrow \downarrow$ \\
Clock Drawing Test (Mendes scoring) & $4 / 10$ & \\
Orientation & & \\
Galveston Orientation Amnesia Test & $68 / 100$ & \\
\hline Attention & & $\mathrm{n}$ \\
Trail Making Test-A & $30 \mathrm{~s}$ & \pm \\
WAIS digit span & 5 & \\
Corsi Test & 4 & $\downarrow$ \\
\hline Executive functions & & \\
Span backward & 2 & $\downarrow \downarrow$ \\
Word fluencies & & \pm \\
F.A.S. & 5 & $\downarrow \downarrow$ \\
Animals & 15 & \\
Colored progressive matrices & 14 & \\
Frontal assessment battery & $9 / 18$ & \\
\hline Anterograde memory & &
\end{tabular}

Anterograde memory

Ray Auditory Verbal Learning Test (15 words)

5 trials (immediate recall) 23/75

5 trials (intrusions) 10

Delayed recall $\quad 5 / 15$

Delayed recognition $\quad 7 / 15$

History memory

$7.5 / 28$

$\downarrow \downarrow$

$\downarrow \downarrow$

Recognition memory (words)

Correct

$24 / 40 \quad \downarrow \downarrow$

Omissions

$16 / 40$

False recognition

$16 / 40$

$\downarrow \downarrow$

Confabulations (Dalla Barba Battery)

Personal semantic memory

Personal episodic memory

I do not know (semantic)

I do not know (episodic)

General semantic memory

Linguistic semantic memory

Orientation

Confabulations

$0 \% \quad \mathrm{n}$

$75 \%$

$50 \%$

$40 \%$

$10 \%$

$0 \%$

$30 \%$

$\downarrow \downarrow$

$\downarrow$

$\downarrow \downarrow$

Visual perception

Drawings copy

10.8/12 n

Poppelreuter overlapping shapes

Montreal-Toulouse agnosia test

$5 / 5$

$20 / 20$

$\mathrm{n}$

Bell Test

5 omissions $\downarrow$ no side

Language

Boston Naming Test

$55 / 60$

Naming on verbal description

$19 / 28$

Token Test

$26 / 36$

$\downarrow$

n

* Corrected for age, sex and educational level. $\mathrm{n}=$ normal; $\pm=$ borderline, $\downarrow=$ impairment, $\downarrow \downarrow$ = severe impairment. 


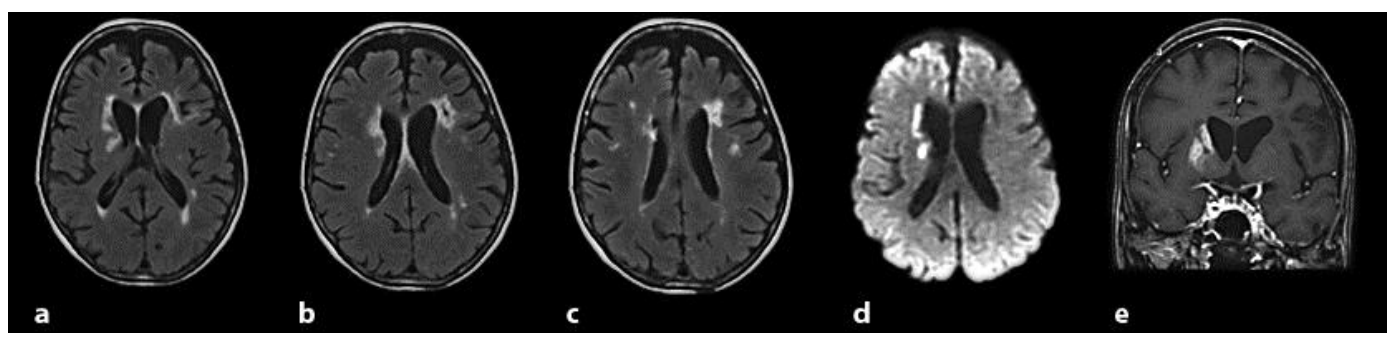

Fig. 1. a-c FLAIR-MRI showing lesions on the right (caudate head and body) and on the left (caudate head and adjacent white matter) lenticulostriate artery territories. $\mathbf{d}$ DWI-MRI shows that only the right lesion is recent. e T1-MRI shows gadolinium enhancement of the lenticular nucleus.

\section{References}

1 Dalla Barba G, Boisse M: Temporal consciousness and confabulation: is the medial temporal lobe 'temporal'? Cogn Neuropsychiatry 2010;15:95-117.

-2 Kopelman MD: Two types of confabulation. J. Neurol Neurosurg Psychiatr 1987;50:1482-1487.

3 Vuilleumier P: Anosognosia; Bogousslavsky J and Cummings JL (eds): Behavior and mood disorders in focal brain lesions. Cambridge University Press, 2000.

4 Feinberg TE, Roane DM: Delusional misidentification. Psychiatr Clin North am 2005;28:665-683.

$\checkmark 5$ Meissner I, Sapir S, Kokmen E, Stein SD: The paramedian diencephalic syndrome: a dynamic phenomenon. Stroke 1987;18:380-385.

6 Schnider A: Spontaneous confabulation and the adaptation of thought to ongoing reality. Nat Rev Neurosci 2003;4:662-671.

7 Dalla Barba G: Different patterns of confabulation. Cortex 1993;29:567-581.

$>8$ McMurtray AM, Sultzer DL, Monserratt L, Yeo T, Mendez MF: Content-specific delusions from right caudate lacunar stroke: association with prefrontal hypometabolism. J Neuropsychiatry Clin Neurosci 2008;20:62-67.

$\$ 9$ Carlesimo GA, Lombardi MG, Caltagirone C: Vascular thalamic amnesia: a reappraisal. Neuropsychologia 2011;49:777-789.

10 Harding A, Halliday G, Kaine D, Kril J: Degeneration of anterior thalamic nuclei differentiate alcoholics with amnesia. Brain 2000;123:141-154.

11 Kopelman MD: Two types of confabulation. J. Neurol Neurosurg Psychiatr 1987;50:1482-1487.

12 Attali E, De Anna F, Dubois B, Dalla Barba G: Confabulation in Alzheimer's disease: poor encoding and retrieval of over-learned information. Brain 2009;132:204-212.

13 Moscovitch M, Melo B: Strategic retrieval and the frontal lobes: evidence from confabulation and amnesia. Neuropsychologia 1997;35:1017-1034.

14 Johnson MK, O'Connor M, Cantor J: Confabulation, memory deficits, and frontal dysfunction. Brain Cogn 1997;34:189-206.

15 Corcoran R: Autonoetic awareness, executive social skills, and the appreciation of intention: figurative reasoning in amnesia, confabulation, and schizophrenia. Cogn Neuropsychiatry 1999;4:55-80.

16 Fotopoulou A: The affective neuropsychology of confabulation and delusion. Cogn Neuropsychiatry 2010;15:38-63.

17 Knowles R, McCarthey-Jones S, Rowse G: Grandiose delusions: a review and theoretical integration of cognitive and affective perspectives. Clin Psychol Rev 2011;31:684-696.

18 Craufurd D, Thompson JC, Snowden JS: Behavioral changes in Huntington Disease. Neuropsychiatry Neuropsychol Behav Neurol 2001;14:219-226.

19 Tsuang D, DiGiacomo L, Lipe H, Bird TD: Familial aggregation of schizophrenia-like symptoms in Huntington's disease. Am J Med Genet 1998;81:323-327. 